\title{
THE BUILDUP OF THE HUBBLE SEQUENCE IN THE COSMOS FIELD
}



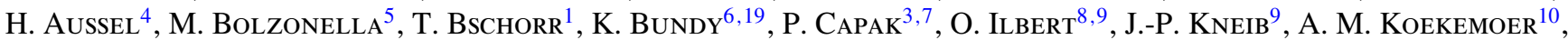 \\ K. Kovač ${ }^{1}$, A. Leauthaud ${ }^{11}$, E. Le Floc' ${ }^{12}{ }^{12}$, R. Massey ${ }^{3,13}$, H. J. McCracken ${ }^{14}$, L. Pozzetti ${ }^{5}$, A. Renzini ${ }^{15}$, J. Rhodes ${ }^{3,16}$,

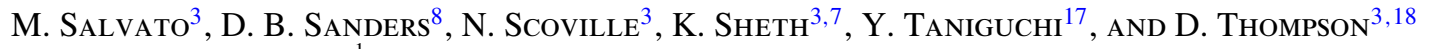 \\ ${ }^{1}$ Institute for Astronomy, ETH Zurich, 8092 Zurich, Switzerland \\ ${ }^{2}$ Max-Planck-Institut für Astronomie, Königstuhl 17, D-69117 Heidelberg, Germany \\ ${ }^{3}$ California Institute of Technology, MS 105-24, 1200 East California Boulevard, Pasadena, CA 91125, USA \\ ${ }^{4}$ AIM Unité Mixte de Recherche CEA CNRS, Université Paris VII UMR n158, Paris, France \\ ${ }^{5}$ INAF-Osservatorio Astronomico di Bologna, Via Ranzani 1, I-40127 Bologna, Italy \\ ${ }^{6}$ Astronomy Department, University of California, Berkeley, CA 94705, USA \\ ${ }^{7}$ Spitzer Space Center, California Institute of Technology, Pasadena, CA 91125, USA \\ ${ }^{8}$ Institute for Astronomy, University of Hawaii, 2680 Woodlawn Drive, Honolulu, HI 96822, USA \\ ${ }^{9}$ Laboratoire d'Astrophysique de Marseille, BP 8, Traverse du Siphon, 13376 Marseille Cedex 12, France \\ ${ }_{10}$ STScI, 3700 San Martin Drive, Baltimore, MD 21218, USA \\ ${ }^{11}$ LBNL \& BCCP, University of California, Berkeley, CA 94720, USA \\ ${ }^{12}$ Service d'Astrophysique, CEA/Saclay, 91191 Gif-sur-Yvette, France \\ ${ }^{13}$ Institute for Astronomy, Royal Observatory, Edinburgh EH9 3HJ, UK \\ ${ }^{14}$ Institut d'Astrophysique de Paris, UMR 7095 CNRS, Université Pierre et Marie Curie, 98 bis Boulevard Arago, 75014 Paris, France \\ ${ }^{15}$ Dipartimento di Astronomia, Universita di Padova, Vicolo dell'Osservatorio 2, I-35122 Padua, Italy \\ ${ }^{16}$ Jet Propulsion Laboratory, MS 169-506, 4800 Oak Grove Drive, Pasadena, CA 91109, USA \\ ${ }^{17}$ Research Center for Space and Cosmic Evolution, Ehime University, Bunkyo-cho 2-5, Matsuyama 790-8577, Japan \\ ${ }^{18}$ LBT Observatory, University of Arizona, 933 North Cherry Avenue, Tucson, AZ 85721-0065, USA \\ Received 2009 November 7; accepted 2010 March 11; published 2010 April 1
}

\begin{abstract}
We use $\sim 8600$ COSMOS galaxies at mass scales $>5 \times 10^{10} M_{\odot}$ to study how the morphological mix of massive ellipticals, bulge-dominated disks, intermediate-bulge disks, disk-dominated galaxies, and irregular systems evolves from $z=0.2$ to $z=1$. The morphological evolution depends strongly on mass. At $M>3 \times 10^{11} M_{\odot}$, no evolution is detected in the morphological mix: ellipticals dominate since $z=1$, and the Hubble sequence has quantitatively settled down by this epoch. At the $10^{11} M_{\odot}$ mass scale, little evolution is detected, which can be entirely explained by major mergers. Most of the morphological evolution from $z=1$ to $z=0.2$ takes place at masses $5 \times 10^{10}-10^{11} M_{\odot}$, where (1) the fraction of spirals substantially drops and the contribution of early types increases. This increase is mostly produced by the growth of bulge-dominated disks, which vary their contribution from $\sim 10 \%$ at $z=1$ to $>30 \%$ at $z=0.2$ (for comparison, the elliptical fraction grows from $\sim 15 \%$ to $\sim 20 \%$ ). Thus, at these masses, transformations from late to early types result in diskless elliptical morphologies with a statistical frequency of only $30 \%-40 \%$. Otherwise, the processes which are responsible for the transformations either retain or produce a non-negligible disk component. (2) The disk-dominated galaxies, which contribute $\sim 15 \%$ to the intermediate-mass galaxy population at $z=1$, virtually disappear by $z=0.2$. The merger rate since $z=1$ is too low to account for the disappearance of these massive disk-dominated systems, which most likely grow a bulge via secular evolution.
\end{abstract}

Key words: galaxies: elliptical and lenticular, $\mathrm{cD}$ - galaxies: evolution - galaxies: formation - galaxies: irregular galaxies: spiral - galaxies: structure

\section{INTRODUCTION}

Over the last decade, astronomers have realized that halo and galaxy masses play a crucial role in galaxy evolution. This has been found both observationally (see, e.g., Kauffmann et al. 2003; Thomas et al. 2005; Baldry et al. 2006; Bamford et al. 2009; Tasca et al. 2009; Cucciati et al. 2009; Bolzonella et al. 2009; Iovino et al. 2009; Kovač et al. 2009) and also theoretically (Hahn et al. 2007a, 2007b, 2009; Skibba \& Sheth 2009; Crain et al. 2009). In particular, early-type galaxies at mass scales $\sim 5 \times 10^{10} M_{\odot}$ progressively increase their contribution to the global galaxy mass function (MF) from $z=1$ down to today, while the most massive early-type galaxies, with masses at and above $\sim 10^{11} M_{\odot}$, are mostly already in place by $z \sim 1$ (e.g., Zucca et al. 2006; Bundy et al. 2006; Franceschini et al. 2006; Caputi et al. 2006; Cimatti et al. 2006; Scarlata

\footnotetext{
${ }^{19}$ Hubble Fellow.
}

et al. 2007a, 2007b; van der Wel et al. 2007; Ilbert et al. 2010; Pozzetti et al. 2009; M. C. Aller et al. 2010, in preparation)

In this Letter, ${ }^{20}$ we push the investigation into the mass growth of massive galaxies one step forward and specifically ask (1) what the detailed morphological mix is, since $z \sim 1$, at all mass scales above $\sim 5 \times 10^{10} M_{\odot}$ (the threshold mass at which $z=0$ galaxies transition between predominantly late- and early-type properties, e.g., Kauffmann et al. 2003) and (2) at what epoch the massive galaxy population is already fully assembled into the final morphological mix that we observe in the $z=0$ Hubble sequence.

Specifically, exploiting the large area and extensive ancillary data sets of the Cosmic Evolution Survey (COSMOS; Scoville et al. 2007a), and the detailed morphological binning provided by the Zurich Estimator of Structural Types (ZEST) classifi-

\footnotetext{
20 Throughout this Letter, we adopt a standard $\Lambda$ CDM cosmology with $\Omega_{M}=0.25, \Omega_{\Lambda}=0.75$, and $h=0.7$. Magnitudes are given in the AB system
} (Oke \& Gunn 1983). 
cation (Scarlata et al. 2007a), we split the $\sim 8600 I_{A B} \leqslant 24$ COSMOS galaxies above a completeness mass cut of $5 \times$ $10^{10} M_{\odot}$ into five separate morphological bins, and we study the evolution of the galaxy morphological mix across the redshift range $z \sim 1-0.2$ as a function of galaxy mass and detailed galactic structure.

\section{DATA, SAMPLE, AND BASIC MEASUREMENTS}

Details on the used data sets, on our mass-complete sample, and on our photometric redshifts and stellar mass estimates, are given in P. A. Oesch et al. (2010, in preparation). Here, we briefly summarize this information. This analysis is based on the Hubble Space Telescope / Advanced Camera for Surveys F814W (I) images of the $1.64 \mathrm{deg}^{2}$ COSMOS field (Scoville et al. 2007b; Koekemoer et al. 2007). In particular, we applied a faint magnitude limit of $I=24$ and a minimum size cut of $r_{1 / 2} \geqslant 0.2$ (with $r_{1 / 2}$ the half-light radius) to the ACS-based catalog of Leauthaud et al. (2007) to ensure a reliable morphological classification. This is based on the ZEST approach described in Scarlata et al. (2007a), which employs a principal component analysis of five measurements of the galaxies' light distribution. These are ellipticity, concentration, asymmetry, the secondorder moment of the $20 \%$ brightest pixels $\left(M_{20}\right)$, and the Gini coefficient. We grouped the galaxies into five classes: (1) E: pure elliptical galaxies (ZEST type 1); (2) B: bulge-dominated disk galaxies. These are the ZEST type 2.0 galaxies, which have non-negligible disk components, but otherwise show global structural properties similar to ellipticals (e.g., concentration, $n$-Sersic values, etc.); (3) $\mathrm{S}$ : spiral galaxies with intermediate bulge properties (ZEST types 2.1 and 2.2 together); (4) D: diskdominated systems (ZEST type 2.3); and, finally, (5) I: irregular galaxies (ZEST type 3) including disturbed merging galaxies. We checked that our results are not biased by misclassification of galaxies at higher redshifts due to the cosmological surface brightness dimming and the smaller apparent sizes of the galaxies.

Our fiducial photometric redshifts are derived using 11 COSMOS passbands, from $u *$ to Spitzer $4.5 \mu \mathrm{m}$, and our Zurich Extragalactic Bayesian Redshift Analyzer $\left(Z E B R A^{21}\right)$ code (Feldmann et al. 2006). The uncertainties in our photo$z$ estimates are $\sigma(z) \sim 0.023(1+z)$ down to $i<22.5$ and $\sigma(z) \sim 0.039(1+z)$ for galaxies between $22.5<i<24$. We also checked the impact of using the 30 band photometric redshifts of Ilbert et al. (2009), which show a smaller dispersion. However, our results remained unchanged when using either photo- $z$ catalog. This is true also for the corresponding stellar mass estimates, which were derived by fitting standard synthetic stellar population model spectral energy distributions (SEDs) to the broadband photometry of each galaxy at its photometric redshift. Our fiducial SED library is based on Bruzual \& Charlot (2003) models following exponentially declining star formation histories reddened by Small Magellanic Cloud dust (Pei 1992; for more details see P. A. Oesch et al. 2010, in preparation). Due to our optical magnitude cut, we are prone to losing red galaxies at higher redshifts. To avoid a possible bias in our results, we conservatively estimate the mass completeness limit of our catalog to be the mass of the model SED with the highest mass-to-light ratio in our template library with a magnitude of $i=24$. The resulting minimum measurable mass at $z=1$ is

\footnotetext{
21 The code is publicly available at the following url:
} www.exp-astro.phys.ethz.ch/ZEBRA.
Table 1

Mass Fraction of Different Types ${ }^{\mathrm{a}}$

\begin{tabular}{crrrrr}
\hline \hline \multirow{2}{*}{ Mass Bin } & Type & \multicolumn{4}{c}{ Redshift Bin } \\
\cline { 3 - 6 } & & \multicolumn{1}{c}{$0.2-0.4$} & \multicolumn{1}{c}{$0.4-0.6$} & \multicolumn{1}{c}{$0.6-0.8$} & \multicolumn{1}{c}{$0.8-1$} \\
\hline \multirow{4}{*}{$5 \times 10^{10}-10^{11} M_{\odot} \odot$} & E & $21.6 \pm 2.9$ & $22.4 \pm 2.8$ & $16.7 \pm 2.0$ & $13.7 \pm 1.6$ \\
& B & $33.3 \pm 4.0$ & $25.0 \pm 3.1$ & $17.7 \pm 2.1$ & $12.4 \pm 1.4$ \\
& D & $1.9 \pm 0.7$ & $4.1 \pm 1.0$ & $9.1 \pm 1.2$ & $15.9 \pm 1.8$ \\
& I & $5.7 \pm 1.2$ & $3.5 \pm 0.9$ & $7.3 \pm 1.0$ & $8.0 \pm 1.0$ \\
\hline \multirow{4}{*}{$M>10^{11} M_{\odot}$} & E & $31.3 \pm 4.2$ & $30.5 \pm 3.8$ & $31.6 \pm 3.5$ & $26.2 \pm 2.9$ \\
& B & $39.4 \pm 5.0$ & $35.0 \pm 4.2$ & $32.1 \pm 3.6$ & $22.6 \pm 2.5$ \\
& S & $26.9 \pm 3.8$ & $30.7 \pm 3.8$ & $29.1 \pm 3.3$ & $38.9 \pm 4.1$ \\
& D & $0.3 \pm 0.5$ & $0.0 \pm 0.3$ & $3.3 \pm 0.7$ & $5.9 \pm 0.9$ \\
& I & $2.1 \pm 1.0$ & $3.8 \pm 1.1$ & $3.9 \pm 0.8$ & $6.4 \pm 0.9$ \\
\hline
\end{tabular}

Note. ${ }^{\text {a }}$ In percent

$M_{\lim }=4.4 \times 10^{10} M_{\odot}$, and in the following analysis we always stay above this limit.

The well-established role of stellar mass in affecting galaxy properties makes it mandatory to separate different mass bins when searching for further evolutionary trends in the galaxy population. In our study, we therefore split our mass-complete sample into two mass intervals, namely, $5 \times 10^{10} M_{\odot}<M<$ $10^{11} M_{\odot}$ and $M>10^{11} M_{\odot}$. The boundaries of these two mass bins are chosen so as to include roughly an equal number of galaxies in each bin and to provide sufficiently robust statistics. The total numbers of galaxies in the bins are 4894 $\left(5 \times 10^{10}-10^{11} M_{\odot}\right)$ and $3707\left(M>10^{11} M_{\odot}\right)$, respectively. We also explore what happens at the very highest mass scales, i.e., for galaxies with $M>3 \times 10^{11} M_{\odot}$ : there are a total of 222 such galaxies in our sample.

\section{THE RISING ROLE OF BULGE-DOMINATED DISK GALAXIES}

The redshift evolution of the mass fraction in different morphological types in our mass bins is plotted in Figure 1 and tabulated in Table 1. Here and in the following, error bars for small number statistics are computed using Gehrels (1986).

At the mass scales and epochs of our study, we note that irregular galaxies are just a minor fraction of the population, which stays remarkably constant at the $<10 \%$ level throughout the $z \sim 0.2-1$ window. Similarly, at these mass scales and epochs, the disk-dominated galaxies provide an almost negligible contribution to the overall galaxy population. Above $\sim 10^{11} M_{\odot}$, their contribution is $<10 \%$ at $z \sim 0.9$, and they essentially disappear by $z \lesssim 0.5$. At the $5 \times 10^{10}<M<10^{11} M_{\odot}$ mass scale, their contribution declines rapidly from $\sim 15 \%$ at $z \sim 0.9$ to a negligible $2 \%$ by $z \sim 0.3$.

At $z \sim 0.9$, the smaller mass bin $\left(5 \times 10^{10}-1 \times 10^{11} M_{\odot}\right)$ is clearly dominated by intermediate-type disk galaxies (left panel of Figure 1), which contribute about $50 \%$ by mass to the whole galaxy population. This fraction decreases gradually toward $\sim 30 \%-40 \%$ by $z \sim 0.3$ which is compensated for by a rising importance of galaxies of earlier morphological types, i.e., by ellipticals and bulge-dominated disk galaxies. At $z \sim 0.9$, these two populations contribute about $10 \%-15 \%$ each to the total galaxy population in this mass bin; by $z \sim 0.3$, these fractions rise to $22 \%$ for the ellipticals and to $33 \%$ for bulge-dominated disk galaxies. We also note that, at these galaxy mass scales, the fraction of stellar mass locked in the elliptical galaxy population is similar to that in bulge-dominated disk galaxies at $z \sim 0.9$. 


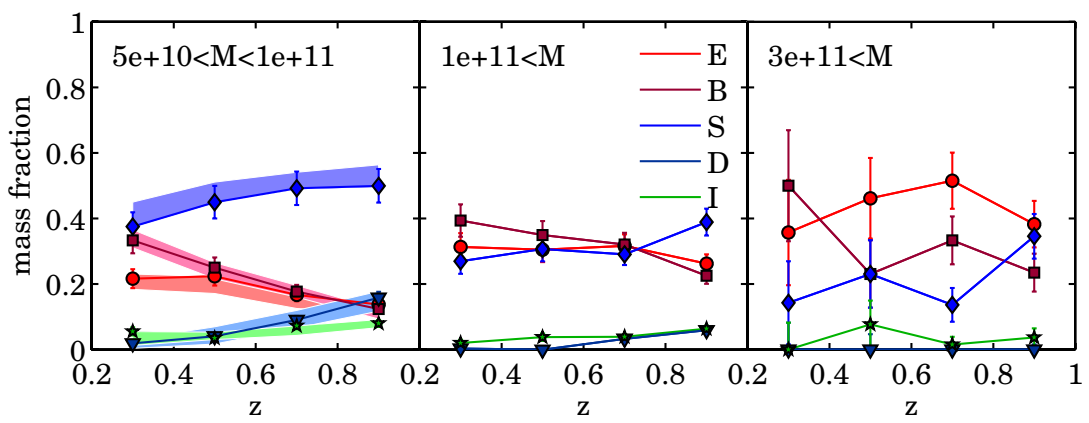

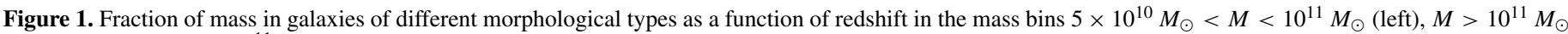

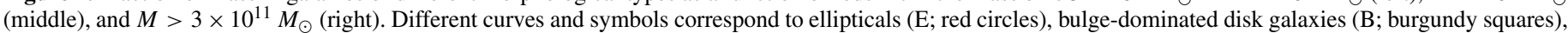

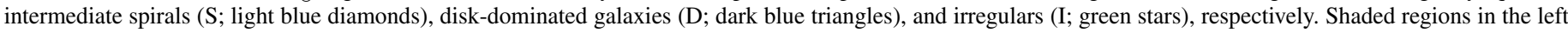

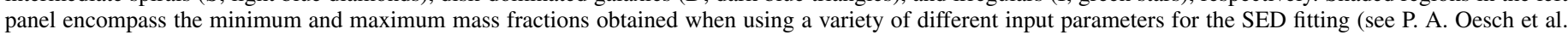
2010, in preparation). Similar uncertainties apply to the other panels and are omitted for clarity.

The latter rises in importance toward lower redshifts much more rapidly than the Es, however, and dominates the mass density of early-type galaxies by redshifts of order $z \sim 0.5$. From the above fractions, we estimate that galaxies above $M>5 \times 10^{10} M_{\odot}$, which transform their morphology into an early-type (either $B$ or $E$ ) between $z=0.9$ and $z=0.3$, have a $\sim 70 \%$ probability of retaining a non-negligible disk (i.e., of becoming $\mathrm{B}$ galaxies).

For galaxy masses above $10^{11} M_{\odot}$ (middle panel of Figure 1), the redshift evolution of the morphological fractions of galaxies of all types is much weaker than at the lower masses. Intermediate-type disk galaxies are still a major contributor to the mass density budget in this bin, but here they dominate only at the highest redshift, where they account for about $40 \%$ of the galaxy population. Early-type galaxies (Es and the bulgedominated B galaxies) contribute larger fractions than at lower masses, both of order $25 \%$ at $z \sim 0.9$, rising up to about $30 \%$ and $40 \%$ by $z \sim 0.3$, respectively.

Within the errors, the highest mass ellipticals $\left(>3 \times 10^{11} M_{\odot}\right)$ maintain their predominance over early types with disk (see the right panel of Figure 1), consistent with detailed analyses of the $z=0$ universe. Overall, not only are early-type galaxies at and above the $M \sim 10^{11} M_{\odot}$ mass scale assembled before their lower mass counterparts, but also their morphological evolution appears to be already stabilized by $z \sim 1$ (see also, e.g., van der Wel et al. 2007; Sheth et al. 2008). The Hubble sequence at the highest masses is not only qualitatively but also quantitatively in place already more than 8 Gyr ago.

\section{THE GROWTH OF MASSIVE GALAXIES SINCE $z=1$}

Possibly the simplest question to ask is whether the morphological distribution can be explained by galaxies growing stellar mass in situ to migrate to a higher mass bin without modifying their morphologies. The simple answer is no, as found by estimating the mass that galaxies with $0.8<z<1$ will achieve if their best-fit star formation histories are extrapolated from the epoch at which they are observed down to $z=0.3$. Internal star formation since $z \sim 0.9$ only results in a very modest increase $(<1 \%)$ in the stellar mass in galaxy types $\mathrm{E}, \mathrm{B}$, and $\mathrm{S}$. Disk-dominated D and irregular galaxies I show, however, a non-negligible mass growth over the time span from $z \sim 0.9$ to $z=0.3$. The median growth for disk-dominated galaxies is $16 \%(9 \%)$ in the lower (higher) mass bin. Also, for galaxy types $\mathrm{E}, \mathrm{B}$, and $\mathrm{S}$, the fraction of galaxies that will have moved from the lower to the higher mass bin by $z \sim 0.3$, solely due to internal star formation, is negligibly small $(\sim 4 \%-7 \%)$. In contrast, a quarter of the disk-dominated and a third of the irregular galaxy population are expected to migrate from the lower to the higher mass bin in the $z \sim 0.9$ to $z \sim 0.3$ time interval. It is particularly interesting that such a large fraction of disk-dominated galaxies (D) is expected to migrate to the higher mass bin by $z \sim 0.3$, given that essentially none of these high-mass objects are found in the COSMOS data. This indicates that the growth of stellar mass in this class of objects must be accompanied by morphological transformations and migration to a different morphological bin.

Physical processes that can move galaxies from a late-type to an early-type morphology range from purely internal evolution to mergers with other galaxies. A first-order quantification of the role of mergers in morphological transformations can be obtained assuming that galaxy morphologies are changed exclusively by merger events in a $\Lambda \mathrm{CDM}$ cosmology. We use the code of Neistein \& Dekel (2008) to generate merger trees over the redshift interval $0.3<z<0.9$ (matching the centers of our observational redshift bins) and populate the corresponding halos with galaxies of given masses and morphologies, in order to trace the redshift evolution of their stellar mass content and morphology. Each halo is populated by only one galaxy (i.e., we neglect substructure for this order of magnitude estimate). The conversion from dark matter halo mass to galaxy stellar mass is computed by matching the cumulative halo MF (Jenkins et al. 2001) with our total galaxy stellar MF, as measured in the COSMOS survey, averaged from $z=0.2$ to $z=1$. This conversion is well fitted by a double power law (see also, e.g., Vale \& Ostriker 2008):

$$
\begin{aligned}
\log M_{g}= & \log M_{0}+a\left(\log M_{\mathrm{DM}}-\log M_{s}\right) \\
& -b \log \left[c+10^{d\left(\log M_{\mathrm{DM}}-\log M_{s}\right)}\right],
\end{aligned}
$$

where $M_{g}$ and $M_{\mathrm{DM}}$ represent the galaxy and the dark matter halo mass, respectively. The best-fit parameters are $\log M_{0}=10.65$, $\log M_{s}=11.40, a=6.90, b=5.13, c=0.73$, and $d=1.27$. Over the mass range of our study, this conversion is in good agreement with the relation between the virial mass of dark matter halos and the stellar mass of the galaxies in the COSMOS mock catalogs of Kitzbichler \& White (2007).

Galaxies are assigned an initial morphological class at the start of the merger tree evolution, i.e., in the highest redshift bin. This is done statistically, according to the measured fractions of morphological types in the given mass bin (Figure 1). As the merger trees evolve, morphological transformations are allowed according to a simple two-parameter model: the first 


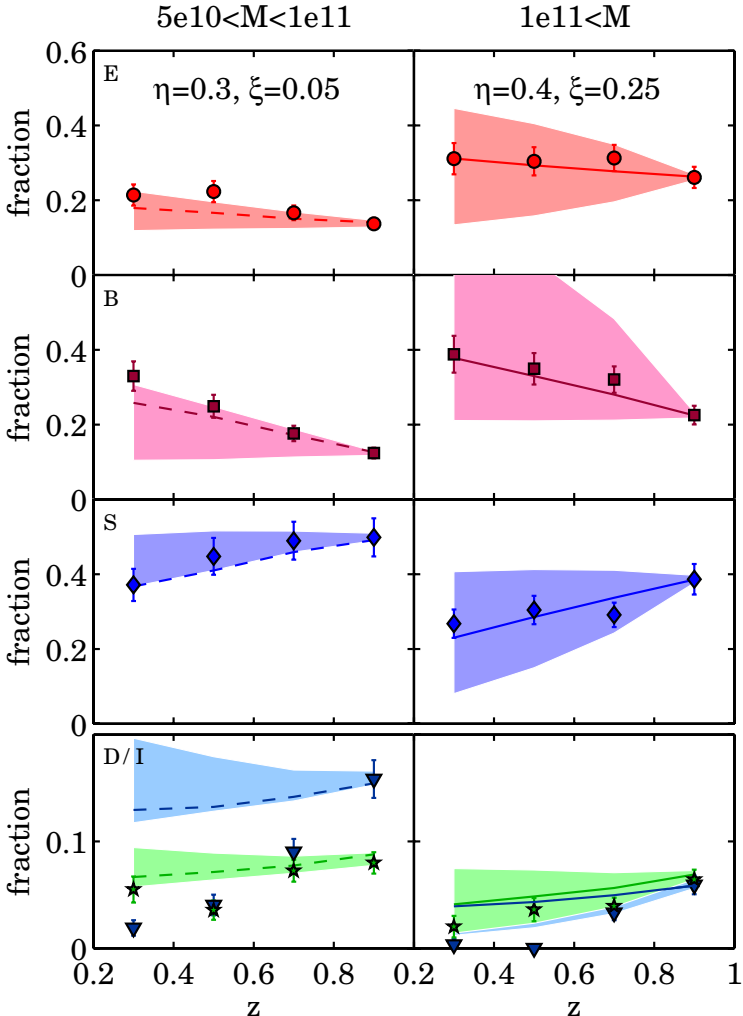

Figure 2. Merger-tree-based models for the morphological evolution in the two different mass bins of all morphological types (note the different plotting scale for $\mathrm{D}$ and I). The shaded areas show all models with parameters in the range $\eta=0.1-0.5$ and $\xi_{\min }=\frac{1}{2}-\frac{1}{20}$, respectively. The data from Figure 1 are shown with large dots and error bars. All models coincide with the data by construction in the highest redshift bin. Evolving toward lower redshifts, galaxies which undergo a merger event acquire an early-type E or B morphology when the stellar mass ratio of their progenitors is larger than $\xi_{\min } . \eta$ is the fraction of such mergers which end up producing an E morphology. Solid lines in the right panels (for $M>10^{11} M_{\odot}$ ) show the self-consistent best-fit solution to all galaxy types in this mass bin, provided by $\xi_{\min }=1 / 4$ and $\eta=0.4$. At lower masses, the best-fit solution (dashed line) would be $\eta=0.3, \xi_{\min }=1 / 20$. However, such low-mass mergers are not expected to induce a significant morphological evolution and additionally, they cannot explain the disappearance of disk-dominated systems (see Figure 3).

parameter sets the smallest mass ratio of merging galaxies which can produce a morphological transformation, $\xi_{\text {min }}$. Specifically, any galaxy which undergoes a merger between two progenitor galaxies with stellar masses in a ratio $>\xi_{\min }$ is attributed either the morphology of a bulge-dominated disk galaxy (B type) or of an elliptical galaxy ( $\mathrm{E}$ type). The probability that such a descendant galaxy is an E galaxy is our second free parameter, $\eta$. We run a large grid of $\eta$ and $\xi_{\text {min }}$ models, encompassing the range of values which are likely to be reasonable for these two uncertain quantities. The results are shown in Figure 2. The shaded areas show the region of parameter space that is covered by the entire range of explored $\xi_{\min }$ and $\eta$ models. In the highest mass bin, a self-consistent best-fit model to all morphological types can be found which is described by $\eta=0.4, \xi_{\min }=\frac{1}{4}$ (solid lines). Thus, in this model, 60\% of all transformations from late to early type result in bulge-dominated galaxies with a non-negligible disk component $(B s)$. The model also reasonably reproduces the evolution of the later D and I galaxy types, as shown in the right panel of Figure 3.

However, no model can reasonably fit the observed data points for all morphological types simultaneously in the lower mass bin (see the left panels of Figures 3 and 2). That is, the models

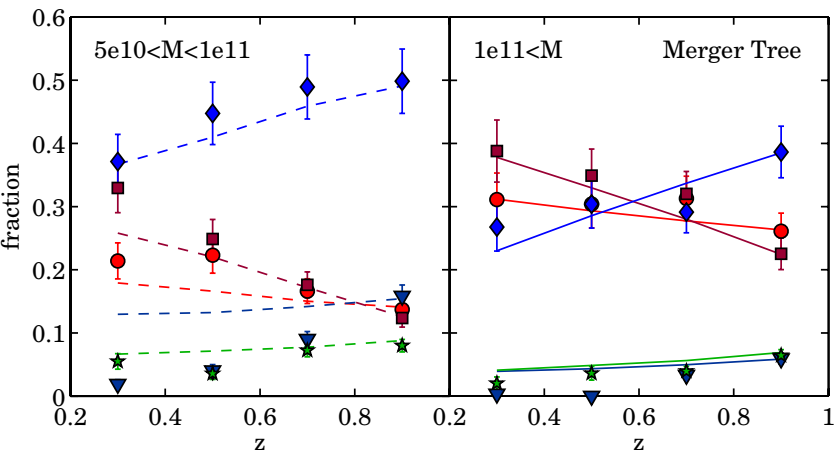

Figure 3. Results of our merger-tree models for all morphological types. Dots with error bars are the measurements shown in Figure 1. The self-consistent evolutionary tracks to all morphological types which best approximate the observed data points are shown as solid/dashed lines in the two different mass bins. Note the failure of the hierarchical models to reproduce the evolutionary trends observed at stellar masses $<10^{11} M_{\odot}$, even with stellar mass ratios of the merging progenitors down to $1 / 20$. Colors and symbols are the same as in Figure 1.

cannot explain the growth in the $\mathrm{E}$ and $\mathrm{B}$ populations and the decline of the later-type populations since $z<0.9$, even when including minor mergers with mass fractions as low as $1 / 20$. One clear feature that is observable in the modeled trends is the increasingly more substantial over-production of diskdominated systems as cosmic time proceeds.

We speculate that, in a hierarchical universe in which mergers are bound to occur, disk-dominated galaxies additionally undergo bulge-building morphological transformations due to internal, secular evolution processes (possibly triggered by the hierarchical accretion activity), such as bar instabilities, gas ejection or stripping. Secular evolution is strongly suggested by many independent studies of the $z=0$ disk galaxy population (see, e.g., Kormendy \& McLure 1993; Norman et al. 1996; Carollo 1999; Carollo et al. 2007, and also Kormendy \& Kennicutt 2004 for a comprehensive list of references). We thus expect secular evolution-driven migration of disk-dominated galaxies into earlier types S (or even B; see also Sargent et al. 2007), which may alleviate the discrepancy between our simple but realistic merger model and our observational results in the COSMOS field.

Note that previous work similarly found the merger rates to be too low to account for the transformations needed to build up early-type galaxies (Genel et al. 2008; Bundy et al. 2007, 2009).

\section{CONCLUSIONS}

Our analysis shows that the evolution of the morphological distribution in galaxies since $z \sim 1$ depends strongly on stellar mass, with little or no evolution at mass scales above $M \sim 10^{11} M_{\odot}$ and detectable evolutionary trends at lower masses $\left(M=5 \times 10^{10}-10^{11} M_{\odot}\right)$.

At $z \sim 1$ between $5 \times 10^{10}$ and $10^{11} M_{\odot}$, there exists a nonnegligible fraction $(\sim 15 \%)$ of disk-dominated galaxies. Their relative abundance decreases rapidly toward lower redshift, essentially disappearing by $z \sim 0$.4. In contrast with all other main types, these systems grow considerable amounts of stellar mass since $z \sim 1$ through internal star formation.

At all masses above our completeness limit, the early-type population grows in importance toward lower redshifts. Interestingly, we find that this growth is dominated by galaxies containing a non-negligible disk component, rather than by pure ellipticals. In the simple-minded framework in which late-type 
galaxies are directly transformed into "early-type" galaxies, about $60 \%-70 \%$ of such transformations result in bulgedominated B-type disk galaxies. The physical mechanisms responsible for these late-to-B transformations must be capable of building or retaining a dynamically cold component, and different mechanisms may be at play in the remaining $\sim 30 \%$ of cases in order to produce the diskless E galaxies.

At mass scales above $\sim 10^{11} M_{\odot}$, a simple but robust mergertree model can reproduce the redshift evolution of the mass fraction of galaxies of all morphological types simultaneously: mergers with stellar mass ratios down to $1 / 4$ can, without any need of invocation of more complex scenarios, reproduce the morphological mix at $z \sim 0.2$ at the high end of the galactic mass scales, where the disk-dominated galaxies are only a negligible contribution.

In contrast, at mass scales below $10^{11} M_{\odot}$, neither the evolution of the fractions of early-type populations ( $\mathrm{E}$ and B) nor that of disk-dominated systems can be reproduced within a $\Lambda C D M$ hierarchical picture including only mergers as the responsible mechanism for morphological transformations. Most prominently, the disappearance of the disk-dominated galaxies is not explained by mergers of any mass ratio, down to small accretion events. Global disk dynamical instabilities are thus likely to be active in building bulge components through inward transfer of mass (see, e.g., Kormendy \& Kennicutt 2004), thereby adding substantial stellar mass while also inducing the galaxy to migrate to an earlier-type morphological class. Secular evolution thus appears to be the key to achieving a complete recipe for the formation of the $z=0$ Hubble sequence at mass scales below $10^{11} M_{\odot}$.

We thank the COSMOS and zCOSMOS collaborations for many stimulating discussions. P.O. acknowledges support from the Swiss National Foundation (SNF). This work is based on observations with the NASA/ESA Hubble Space Telescope, obtained at the Space Telescope Science Institute, which is operated by AURA Inc, under NASA contract NAS 5-26555; also based on data collected at: the Subaru Telescope, which is operated by the National Astronomical Observatory of Japan; the European Southern Observatory under Large Program 175.A-0839, Chile; Kitt Peak National Observatory, Cerro Tololo Inter-American Observatory, and the National Optical Astronomy Observatory, which are operated by the Association of Universities for Research in Astronomy, Inc. (AURA) under cooperative agreement with the National Science Foundation; and the Canada-France-Hawaii Telescope with MegaPrime/ MegaCam operated as a joint project by the CFHT Corporation,
CEA/DAPNIA, the National Research Council of Canada, the Canadian Astronomy Data Centre, the Centre National de la Recherche Scientifique de France, TERAPIX, and the University of Hawaii.

\section{REFERENCES}

Baldry, I. K., et al. 2006, MNRAS, 373, 469

Bamford, S. P., et al. 2009, MNRAS, 393, 1324

Bolzonella, M., et al. 2009, A\&A, submitted (arXiv:0907.0013)

Bruzual, G., \& Charlot, S. 2003, MNRAS, 344, 1000

Bundy, K., et al. 2006, ApJ, 651, 120

Bundy, K., et al. 2007, ApJ, 665, 5

Bundy, K., et al. 2009, ApJ, 697, 1369

Caputi, K., et al. 2006, MNRAS, 366, 609

Carollo, C. M. 1999, ApJ, 523, 566

Carollo, C. M., et al. 2007, ApJ, 658, 960

Cimatti, A., et al. 2006, A\&A, 456, L29

Crain, R. A., et al. 2009, MNRAS, 399, 1773

Cucciati, O., et al. 2009, A\&A, submitted

Feldmann, R., et al. 2006, MNRAS, 372, 565

Franceschini, A., et al. 2006, A\&A, 453, 397

Gehrels, N. 1986, ApJ, 303, 336

Genel, S., et al. 2008, ApJ, 688, 789

Hahn, O., et al. 2007a, MNRAS, 375, 489

Hahn, O., et al. 2007b, MNRAS, 381, 41

Hahn, O., et al. 2009, MNRAS, 398, 1742

Ilbert, O., et al. 2009, ApJ, 690, 1236

Ilbert, O., et al. 2010, ApJ, 709, 644

Iovino, A., et al. 2010, A\&A, 509A, 40

Jenkins, et al. 2001, MNRAS, 321, 372

Kauffmann, G., et al. 2003, MNRAS, 341, 54

Kitzbichler, M. G., \& White, S. D. M. 2007, MNRAS, 376, 2

Koekemoer, A., et al. 2007, ApJS, 172, 196

Kormendy, J., \& Kennicutt, R. C. 2004, ARA\&A, 42, 603

Kormendy, J., \& McLure, R. D. 1993, AJ, 105, 1793

Kovač, K., et al. 2010, ApJ, in press (arXiv:0909.2032)

Leauthaud, A., et al. 2007, ApJS, 172, 219

Neistein, E., \& Dekel, A. 2008, MNRAS, 383, 615

Norman, C. A., et al. 1996, ApJ, 462, 114

Oke, J. B., \& Gunn, J. E. 1983, ApJ, 266, 713

Pei, Y. C. 1992, ApJ, 395, 130

Pozzetti, L., et al. 2009, A\&A, submitted (arXiv:0907.5416)

Sargent, M., et al. 2007, ApJS, 172, 434

Scarlata, C., et al. 2007a, ApJS, 172, 406

Scarlata, C., et al. 2007b, ApJS, 172, 494

Scoville, N., et al. 2007a, ApJS, 172, 1

Scoville, N., et al. 2007b, ApJS, 172, 38

Sheth, K., et al. 2008, ApJ, 675, 1141

Skibba, R. A., \& Sheth, R. K. 2009, MNRAS, 392, 1080

Tasca, L., et al. 2009, A\&A, 503, 379

Thomas, D., et al. 2005, ApJ, 621, 673

Vale, A., \& Ostriker, J. P. 2008, MNRAS, 383, 355

van der Wel, A., et al. 2007, ApJ, 670, 206

Zucca, E., et al. 2006, A\&A, 455, 879 